\title{
GPPS-CH-2020-0139
}

\section{EFFECT OF DIFFUSER INHOMOGENEITY ON FULL-ANNULUS AXIAL TURBINE PERFORMANCE}

\author{
Marcel Oettinger, Michael Henke, Joerg R. Seume \\ Institute of Turbomachinery and Fluid Dynamics \\ Leibniz Universität Hannover \\ oettinger@tfd.uni-hannover.de \\ Garbsen, 30823, Germany
}

\begin{abstract}
Gas-turbine stacks are typically designed to be as short as possible, often featuring an aggressive diffuser and an exit plenum with a radial bend in order to reduce transmission shaft length. This can, however, cause flow inhomogeneity at the turbine outlet affecting performance. In this paper, the effect of such inhomogeneity is investigated utilizing experimentally supported full-annulus simulations of the turbine and exhaust sections. It is demonstrated that an inhomogeneous static pressure distribution can cause variation in upstream blade loading of up to $1.3 \%$ in the Zweifel coefficient. This effect becomes stronger for higher diffuser inlet swirl angles, stressing the importance of interlocked turbine-exhaust section design.
\end{abstract}

\section{INTRODUCTION}

In order to minimize power-shaft length and accommodate torque transmission to the generator, the exhaust section of gas turbines is usually designed to be as short as possible. This design can be realized in two major ways: First, the length of the annular diffuser section is kept as short as possible, resulting in the diffuser not fully expanding the gas. Secondly, the exhaust plenum is usually executed as an exit casing with a sharp bend, which allows for positioning the gear box as close to the shaft bearing as possible.

Such short-stack configurations are typical for power plants, but also commonly found in research turbines, where the maximum size is often limited. One drawback of such a configuration is a potential aerodynamic influence on the outlet flow field as the exit casing and the sharp bend in particular, cause a variation in flow-path length across the circumference. Turbine inhomogeneity is commonly associated with upstream causes, particularly the combustion chamber (Pyliouras et al., 2012). Previous research has focused on hot-streak propagation
(Basol et al., 2011) and the distortion influence on aeroelastic properties (Simonassi et al., 2019). An inhomogeneous pressure field induced by the exit stack, in turn, could similarly affect turbine performance, as disturbances can be transported upstream through the diffuser and act as an outlet boundary condition on the turbine.

Great efforts have been made for understanding the effect of the turbine outlet flow field on diffuser performance, particularly that of tip jets induced upstream of the diffuser. Numerous authors, such as Babu et al. (2011) and Volkmer et al. (2011) found an increase in diffuser pressure recovery for increasing jet intensity. Mimic et al. (2017) were able to correlate the diffuser pressure recovery with stage parameters of the blading, demonstrating a direct correlation between the intensity of tipgap vortices and boundary layer stabilization for an engine-like test rig. Unlike secondary flows, Opilat (2011) found blade wakes entering the diffuser to only have a small influence on diffuser performance.

Additionally, modern gas-turbine requirements demand a increasingly broad operating range with more frequent load changes. High swirl angles typically worsen diffuser performance, as they increase flow path length and can induce flow separation as demonstrated experimentally by Vassiliev et al. (2008) and Pradeep et al. (2010). Higher swirl angles are especially common in part-load operation (Opilat (2011)), which is thus associated with worse diffuser performance as shown by Chernikov (2008). In some cases, moderate swirl angles were found to be beneficial (Vassiliev et al., 2011). It is unclear, however, whether this is due to the swirl itself being beneficial or due to higher stabilization due to more intensive tip-gap vortices. Struts in the diffuser inlet section can be beneficial through diminishing swirl and its adverse effect, see Feldcamp and Birk (2006) and Fleige et al. (2002). 
While the literature regarding turbine-diffuser interaction is extensive, the inverse impact of diffuser-induced disturbances on turbine performance has been investigated to a lesser degree. Moser (1989) investigated experimentally the effect of turbine outlet pressure distortions and found the distorted flow field to excite the blading. Similar results were found for radial turbines and impellers by Sideris and Van den Braembussche (1987).

The aim of this work is assessing diffuser inlet inhomogeneities, investigating their cause and evaluating their impact on the flow field of the upstream turbine. For this investigation, a partial full-annulus turbine setup is derived and evaluated. An investigation of the diffuser inlet flow state forms the basis for analyzing flow deviation in circumferential direction. Additional simulations of the diffuser-exhaust-plenum section finally enable a sensitivity analysis of flow inhomogeneity caused by variations in operating point and diffuser inlet swirl angle. The objectives of this work can be summarized with the following questions:

1. How does an exit casing with a 90 deg bend affect the turbine outlet flow state?

2. What is the resulting effect on turbine performance?

3. How does the observed distortion depend on operating state and swirl angle?

\section{Characteristic parameters}

In order to evaluate turbine and diffuser performance, several non-dimensional parameters are used. All static and total pressure results are related to the state at diffuser inlet, i.e.,

$$
p_{\text {rel }}=\frac{p-p_{\mathrm{D}, \text { in }}}{p_{\mathrm{t}, \mathrm{D}, \mathrm{in}}-p_{\mathrm{D}, \text { in }}}
$$

and

$$
p_{\mathrm{t}, \mathrm{rel}}=\frac{p_{\mathrm{t}}-p_{\mathrm{D}, \mathrm{in}}}{p_{\mathrm{t}, \mathrm{D}, \mathrm{in}}-p_{\mathrm{D}, \mathrm{in}}} .
$$

In order to assess blade loading, the Zweifel coefficient

$$
\mathrm{ZW}=\frac{\int_{\mathrm{LE}}^{\mathrm{TE}}\left(p_{\mathrm{PS}}-p_{\mathrm{SS}}\right) \mathrm{d}\left(\frac{x}{C_{\mathrm{ax}}}\right)}{p_{\mathrm{t}, \mathrm{LE}}-p_{\mathrm{TE}}}
$$

which relates the integral pressure difference between pressure and suction side to the dynamic pressure head between leading and trailing edge, is used. Diffuser performance can be characterized using the total pressure loss coefficient

$$
\zeta=\frac{p_{\mathrm{t}, \mathrm{D}, \text { in }}-p_{\mathrm{t}, \mathrm{D}, \text { out }}}{p_{\mathrm{t}, \mathrm{D}, \mathrm{in}}-p_{\mathrm{D}, \mathrm{in}}}
$$

and the pressure recovery factor

$$
c_{p, \mathrm{D}}=\frac{p_{\mathrm{D}, \mathrm{out}}-p_{\mathrm{D}, \mathrm{in}}}{p_{\mathrm{t}, \mathrm{D}, \mathrm{in}}-p_{\mathrm{D}, \mathrm{in}}} .
$$

In order to assess flow inhomogeneity at the turbine outlet a distortion factor

$$
\mathrm{DF}_{\theta, \text { max }}=\max \left[\frac{p(\theta)}{\bar{p}}\right]
$$

is used. A segment of $\theta=10 \mathrm{deg}$ is chosen, as this is equal to one blade pitch of the last turbine stator row. We assess the maximum passage pressure in relation to the pressure averaged across the entire turbine outlet. With the exception of static pressure-which is area-averaged-all flow variables are massaveraged across the specified planes.

\section{TEST CASE}

The turbine configuration investigated and the numerical setup used are detailed in the following section.

\section{Turbine configuration}

The present configuration is a 1.5-stage low-pressure turbine featuring an annular diffuser of comparably short length and an exit casing with a 90 deg bend immediately downstream of the diffuser exit. Since the final stator row induces considerable swirl even at design point, it is particularly suited for this investigation. As depicted in Fig. 1, the transition between diffuser and and exit casing is discontinuous, causing flow separation at the exit casing inlet. The rotating part of the hub wall extends almost to the diffuser inlet. Cavities separate the stators from the rotor shaft. The rotor is shrouded with the shroud describing a similar geometry as the hub-side cavities.

The isolated turbine configuration has been the subject of several studies, e.g., Henke et al. (2016) and Oettinger et al. (2019b), where both the geometrical parameters and aerodynamic characteristics are described in detail, of which a summary is given in Tab. 1 . The axial gap is adjusted to $50 \%$ of the stator axial chord.

\section{Table 1 Test conditions and stage characteristics at} design point (Oettinger et al., 2019b)

\begin{tabular}{llll}
\hline Rotational speed $n$ in $1 /$ min & & 6950 & \\
Inlet total pressure $p_{\mathrm{t} \text {,in }}$ in $\mathrm{kPa}$ & & 168.6 & \\
Inlet total temperature $T_{\mathrm{t}, \text { in }}$ in $\mathrm{K}$ & & 344.2 & \\
Mass flow rate $\dot{m}$ in $\mathrm{kg} \mathrm{s}^{-1}$ & & 8.9 & \\
Total pressure ratio $\Pi_{\mathrm{t}}$ & & 1.42 & \\
\hline & Stator 1 & Rotor & Stator 2 \\
Number of blades & 18 & 30 & 36 \\
Aspect ratio AR & 2.15 & 2.15 & 2.15 \\
Reynolds number Re & $7.5 \cdot 10^{5}$ & $6.5 \cdot 10^{5}$ & $4.2 \cdot 10^{5}$ \\
\hline
\end{tabular}




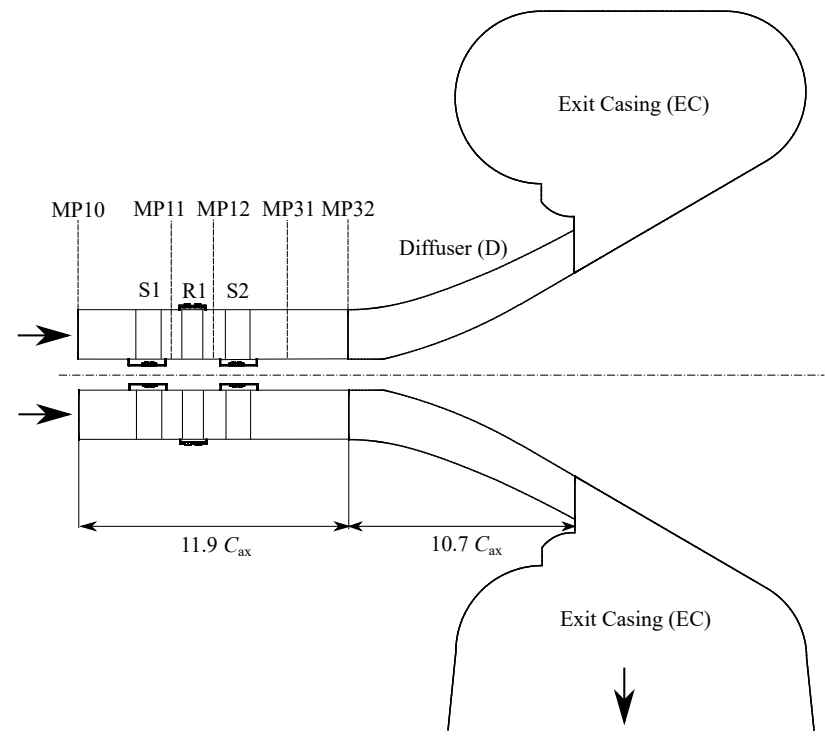

Figure 1 Schematic cross-section of turbine configuration including exit section

\section{Instrumentation}

Extensive time-averaged and time-accurate measurements were conducted within the turbine itself, see Henke et al. (2016) and Oettinger et al. (2019a). Six Struts at the turbine inlet feature total pressure instrumentation (kiel-head probes) and thermocouples at the leading edge with six radial positions each. Henke et al. (2016) demonstrated that the turbine inflow is largely homogeneous without swirl. When analyzing inhomogeneities, we can therefore preclude external causes upstream of the turbine; all flow inhomogeneities observed must therefore be caused within the described control volume.

For the following study, the instrumentation at the final row and inside the diffuser is most relevant and will be detailed below. The stator 2 blading features steady and unsteady pressure taps delivering the profile pressure distribution. Downstream of the stator 2 trailing edge (MP31) pneumatic five-hole probe traverses were conducted as well. The diffuser inlet features five equally spaced rake probes which are instrumented with kiel-head probes and thermocouples measuring the total state at the outlet. Additionally, at several locations three-hole probes replace the kiel-head probes, delivering information on static exit pressure and flow angle. As shown in Fig. 2, only the three-hole probe at $57 \%$ channel height is captured redundantly across the circumference, all other radial positions only exist at discrete circumferential positions. Kiel-head and three-hole probes are twisted to align with the expected flow angle at the design point of the turbine. The diffuser inlet and the diffuser section itself also feature static pressure taps at the casing. At the diffuser inlet (MP32), five wall taps repeat the circumferential spacing of the rakes. In axial direction, ten equidistant wall taps measure the pressure distribution at $90 \mathrm{deg}$ and at $270 \mathrm{deg}$. The measurement uncertainty for wall taps, either on the blading or at the casing, is equal to $85 \mathrm{~Pa}$ while that of three-hole probes equals $250 \mathrm{~Pa}$.

\section{Numerical Setup}

The TRACE solver (Franke et al., 2005), which is being developed by the German Aerospace Center (DLR) in cooperation with MTU Aero Engines AG, is used for steady-state calculations. For the turbine section, details regarding the numerical setup and grid can be found in Oettinger et al. (2019b). Turbine and diffuser consist of structured meshes, the exit casing is described by an unstructured mesh with 34,393,070 grid cells due to its more complex geometry. The structured mesh is resolved by 117 cells in the radial direction with a growth rate of 1.2 at the end walls. The same growth rate is applied to the unstructured domain, where the minimum element size at the walls is equal to $1 \mathrm{~mm}$. The exit-casing walls and the walls inside the cavities are not resolved; instead, wall functions are used locally. For all resolved walls $y^{+} \leq 1$ is adhered to, while for the unresolved walls $y^{+}=10 \ldots 30$. All walls are assumed to be adiabatic. For the structured domain, a grid independence analysis on a mesh featuring a similar topology to the one used in this study is reported in Biester et al. (2012). The full-annulus setup described below totals 224,311,305 cells.

A second-order accurate Fromm scheme (Darwish, 1993) using the van Albada limiter (van Albada et al., 1982) is used for spatial discretisation. Viscous fluxes are solved using a second-order accurate central difference scheme. Turbulence is modeled using the Wilcox $1988 k$ - $\omega$-model (Wilcox, 1998) with the correction by Kato and Launder (1993) to correct the turbulent kinetic energy production near the stagnation point. Rotational effects are accounted for using the extension by Bardina et al. (1985). A multi-mode transition model as described by Kožulović et al. (2007) is used to model transition at the blading. The full-annulus calculation converged to a maximum residual of $L_{\infty}=7.38 \times 10^{-3}$ and a mean residual of $L_{1}=5.98 \times 10^{-8}$.

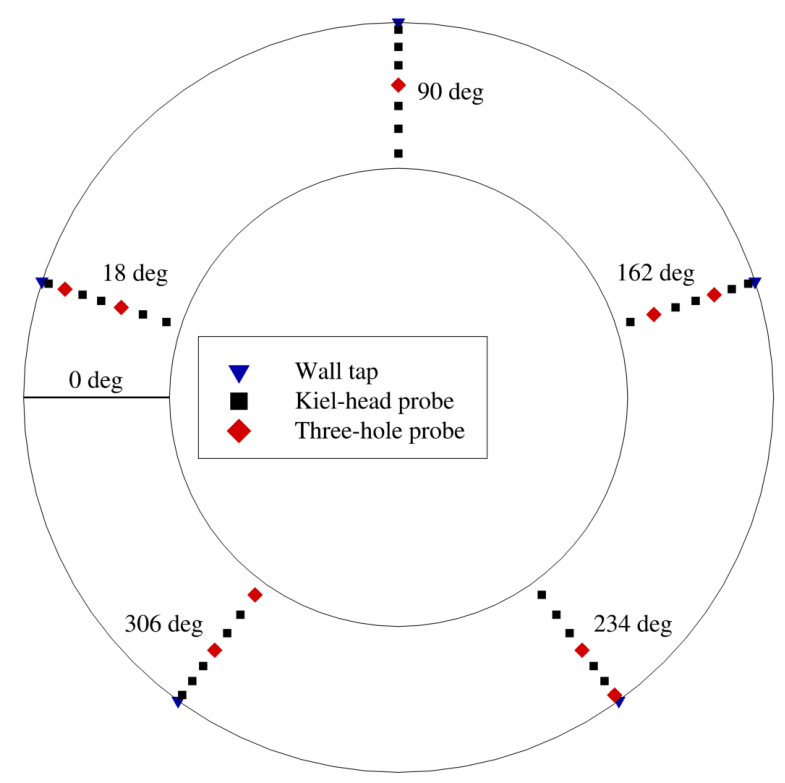

Figure 2 Instrumentation at turbine outlet / diffuser inlet (MP32) 

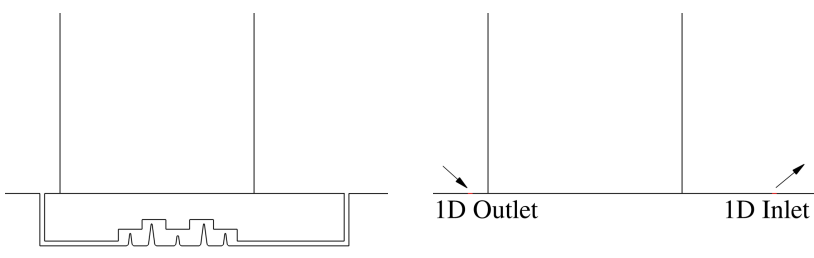

\section{Figure 3 Schematic depiction of cavity simplification}

While stator 1 and the rotor are always calculated as singlepassage models, the second stator domain and the diffuser can be extended to full-annulus configurations. Mixing planes are utilized at $50 \%$ of the axial gap between stator 1 and the rotor as well as between the rotor and stator 2. For full-annulus simulations, zonal interfaces are used for all other interfaces, i.e., at the diffuser inlet and between diffuser and exit casing. Upstream wakes and downstream disturbances can therefore be convected across these interfaces. On the other hand, disturbances originating at the diffuser and exit casing cannot be convected upstream beyond stator 2 , as all circumferential inhomogeneities are mixed out at the mixing plane between the rotor and stator 2 .

In order to enable full-annulus simulations, some simplifications compared to the reference model are necessary. Because of their considerable impact on computational performance, the stator 2 hub-side cavity is neglected in the full-annulus model. As per Fig. 3, the cavity geometry is removed and the interfaces between the cavity and main-flow domains are replaced by an outlet at the leading edge and an inlet at the trailing edge. Leakage flow leaving and entering the main flow path at the end wall is thus directly prescribed in the numerical model. The flow variables specified, i.e. mass-flow rate, total pressure and

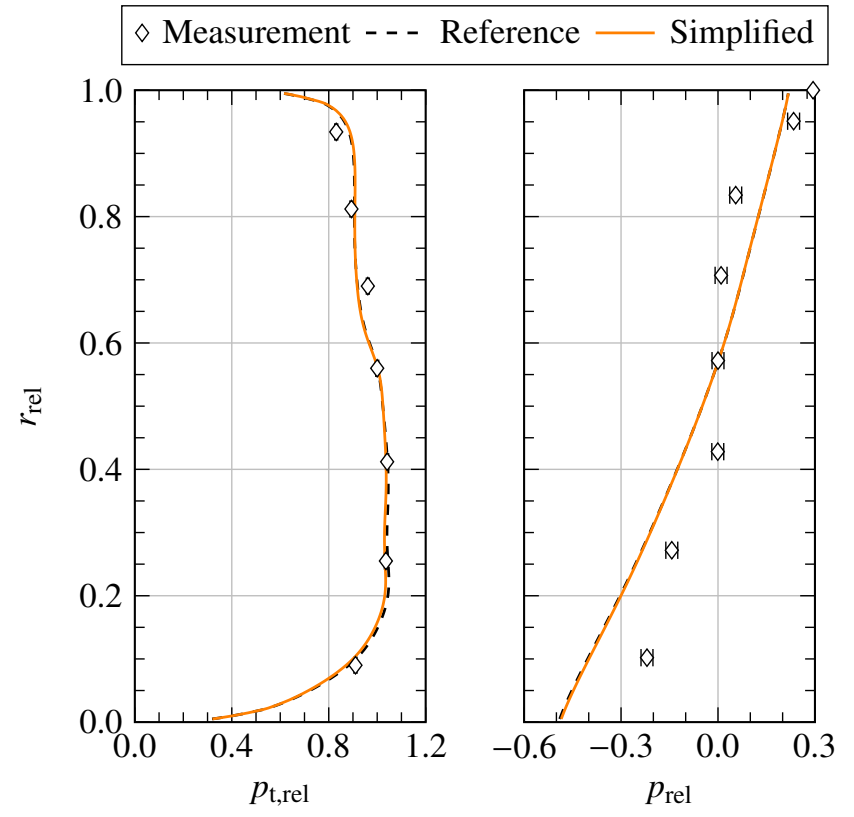

Figure 4 Total pressure (left) and static pressure (right) at turbine outlet (MP32) $\diamond$ Measurement - - - Reference — Simplified

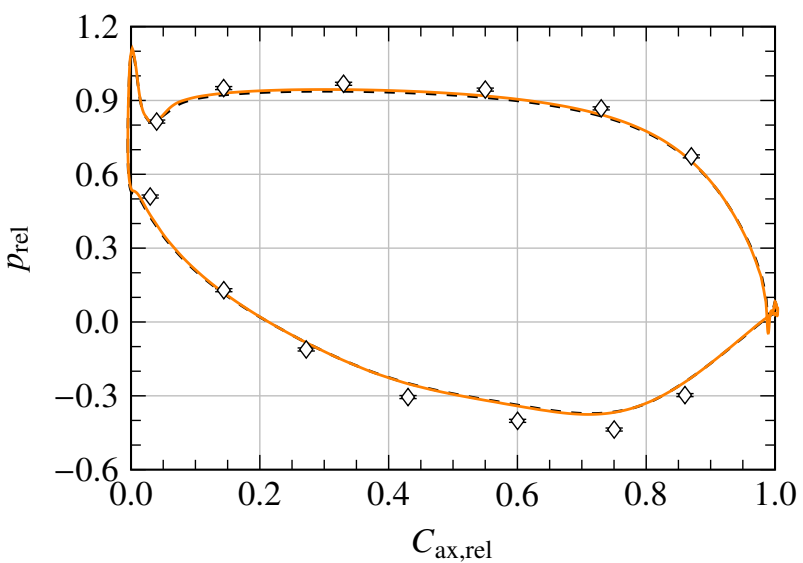

Figure 5 Comparison of stator 2 pressure distribution at mid-span

temperature, flow direction and turbulence quantities, are obtained from the reference calculation including cavities. Since the turbine operating point is never varied, this simplification is valid for all calculations conducted in this study.

As depicted in Fig. 4, the cavity simplification has little impact on the numerical results. It is important to note that due to the normalization chosen, pressures will always agree at midspan. Nevertheless, the experimental trend of the total pressure distribution is well reproduced by the numerical models. For the static pressure distribution, larger differences exist, particularly in the hub region. Only the positions at midspan and at the casing are resolved by several measurement positions across the circumference. As described in Fig. 2, all other measurements are obtained at singular circumferential positions. No clear trend is apparent in the experiment, where the pressure is at some positions higher and at some positions lower compared to the numerical simulation, i.e. the calculated circumferential average. This discontinuous outlet distribution could therefore indicate a circumferential variation in static pressure.

As another simplification, transition at the second stator is neglected to reduce computational effort, due to the boundary layer being almost fully turbulent. As can be seen in Fig. 5, this assumption is valid at the second stator where both calculations produce identical results and agree well with the measurements.

Table 2 Numerical model overview

\begin{tabular}{lll}
\hline Model & Resolved Passages & Interfaces \\
\hline Reference & Single passage & Mixing Planes \\
Simplified & Single passage & Mixing Planes \\
Full-annulus & Single passage (S1,R) / & Mixing Planes / \\
& Full annulus (S2,D,EC) & Zonal \\
Full-annulus with- & Single passage (S1,R) / & Mixing Planes / \\
out exit casing & Full annulus (S2,D) & Zonal \\
Exit stack & Full annulus (D,EC) & Zonal \\
\hline
\end{tabular}


Table 2 gives an overview of all numerical setups used in this study. The full-annulus model is derived from the simplified model discussed above by expanding the stator 2 domain and including the diffuser and exit-casing domains. From this full-annulus configuration two reduced configurations are then derived: One configuration including turbine and diffuser section, but not the exit casing, and a configuration consisting of only the exit stack, i.e. diffuser and exit casing. The latter model is used to investigate additional diffuser inlet swirl angles. For this configuration, an averaged inlet boundary condition is derived from the full-annulus calculation; the diffuser inlet swirl angle is then independently varied while all other operating parameters (inlet total state, mass flow rate) remain constant. This allows for determining the characteristic behavior of flow inhomogeneities with regard to the swirl angle.

\section{FULL-ANNULUS RESULTS \\ Turbine outlet flow field}

In the following section, results derived from the fullannulus calculation are evaluated. We initially consider the total pressure distribution at diffuser inlet (MP32). Fig. 6 shows that the total pressure distribution remains the same for all passages across the circumference even if the exit casing is included in the numerical calculation. Deviations inside the passages, caused by the stator 2 wakes, secondary flows and end-wall boundary layers are visible and show no variation between individual passages. The total temperature distribution is also identical for all passages (not shown here). Since the

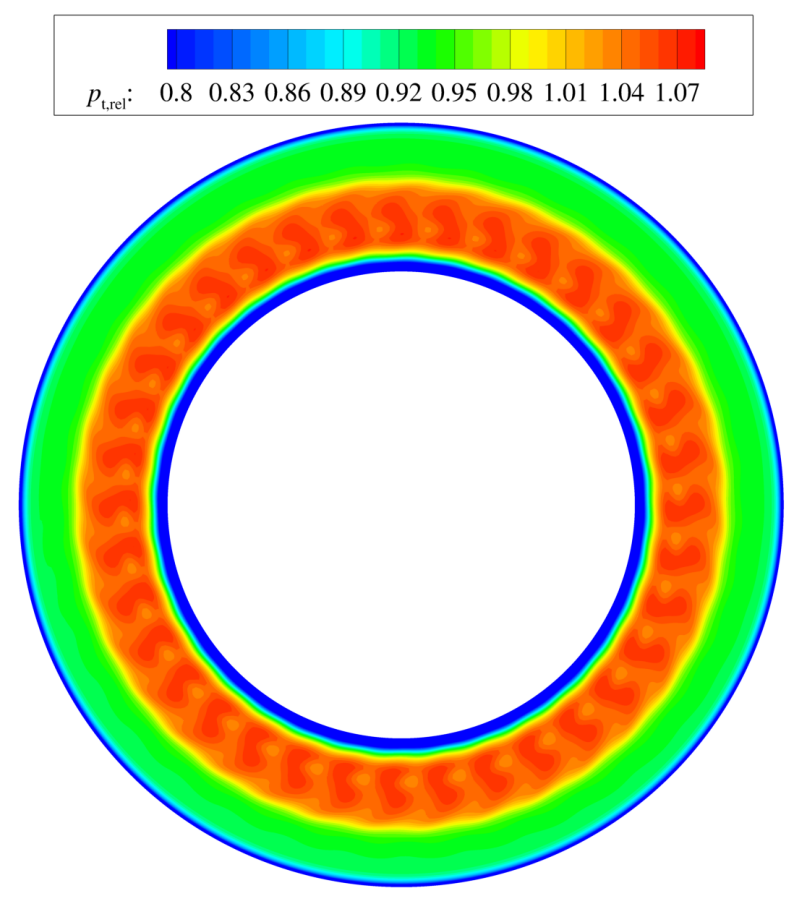

Figure 6 Two-dimensional total pressure distribution at diffuser inlet (MP32)

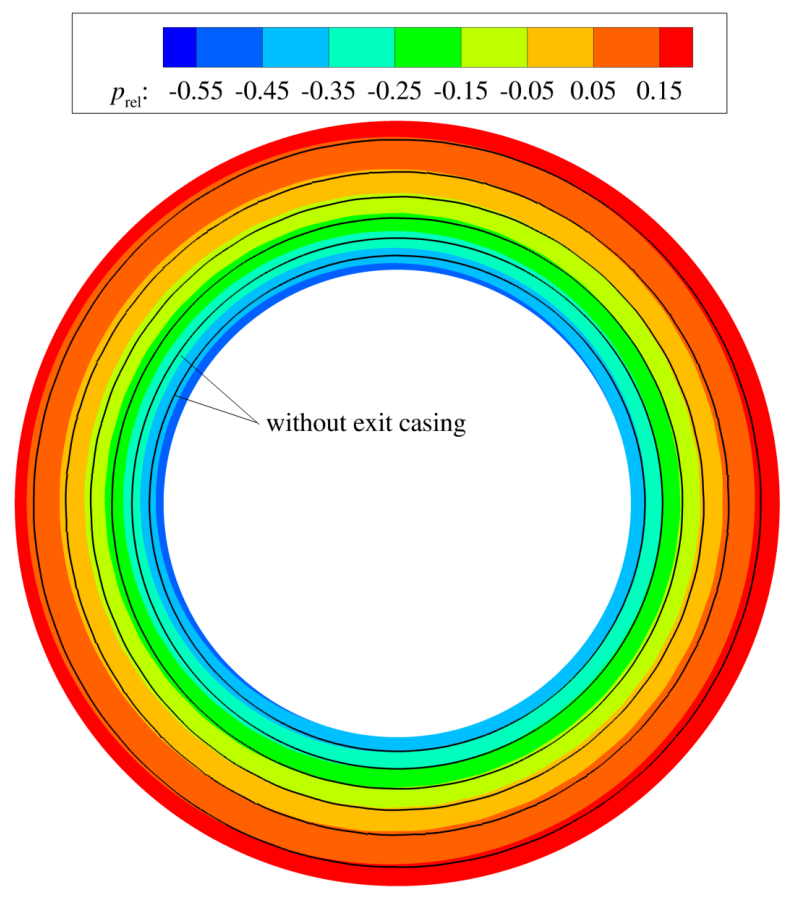

Figure 7 Two-dimensional pressure distribution at
diffuser inlet (MP32). Black contour lines indicate
model without exit casing

turbine inlet flow field is homogeneous in nature and because a mixing plane separates the rotor from the downstream region, no potential flow disturbances can affect the work extraction process.

Unlike the total pressure distribution, the static pressure distribution shows a variation across the circumference (Fig. 7). The pressure in the top left flow region is lower than in the bottom right region. As indicated by the additional pressure contour lines, if the exit casing is neglected in the reduced fullannulus model consisting only of turbine and diffuser sections, the outlet flow field remains entirely homogeneous with regard to circumferential position. It can therefore be concluded that the observed circumferential variation is indeed induced by the exit casing. The diffuser has no direct influence on this flow inhomogeneity, but rather links the circumferentially varying back-pressure induced by its interaction with the exit casing to the upstream turbine section.

Considering the axial pressure distribution at the casing inside the diffuser (Fig. 8), similar results are visible. The pressure at the $270 \mathrm{deg}$ position is higher than at the $90 \mathrm{deg}$ position across the entire diffuser length. While an offset between numerical results and experimental data is apparent, the measurements do confirm this trend, i.e., a pressure variation correlating with circumferential position. The differences in experimental and calculated pressure rise could be the result of an error in the numerical prediction of diffuser flow separation behavior. For the calculation, the deviation between both positions shows an increase with increasing diffuser length. 
Assuming deviations to be highest immediately at the discontinuous transition between diffuser and exit casing, it is apparent that these disturbances increasingly mix out when transported upstream. In the experimental data, however, the deviation magnitude remains largely the same along the axial coordinate.
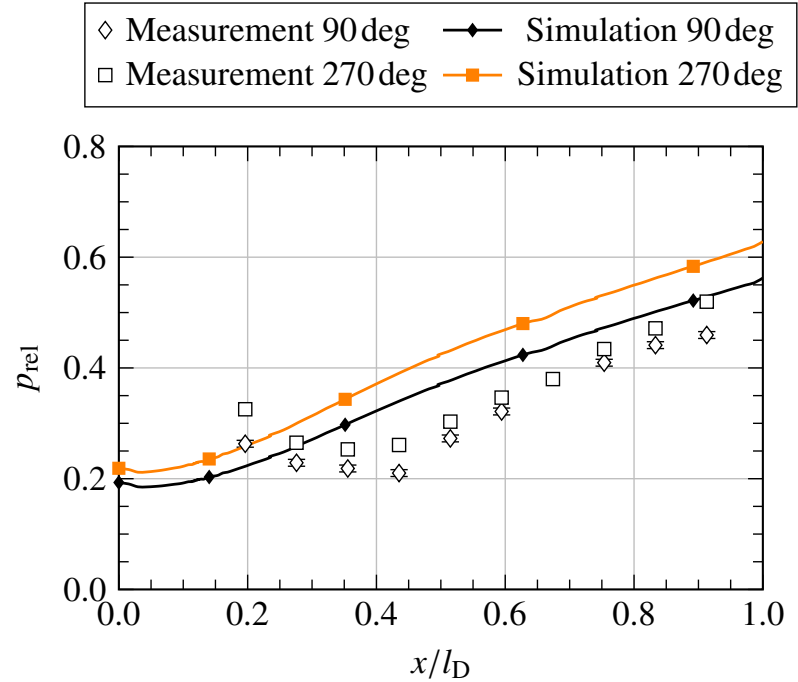

Figure 8 Axial pressure distribution in diffuser section

A quantitative analysis of flow field variation is depicted in Fig. 9 including the equidistantly spaced casing wall taps in MP32. As was already observed in the circumferential average at the casing (Fig. 4), the casing-pressure magnitude is underpredicted by the numerical calculation. With the exception of the measurement position at $162 \mathrm{deg}$, the experiment, however, shows a similar variation across the circumference. The singular measurement at $162 \mathrm{deg}$ deviates by almost $1 \%$ from the circumferential average-more than any other positionwhich might indicate a local measurement error. This, however, cannot be confirmed with absolute certainty. Also indicated are the locations of three-hole probe measurements at their corresponding circumferential and radial positions.

We can note that the measurement positions at $\left(r_{\text {rel }} \theta\right)=$ $(0.10 \mid 306),\left(r_{\text {rel }} \mid \theta\right)=(0.27 \mid 162)$ and $\left(r_{\text {rel }} \mid \theta\right)=(0.95 \mid 243)$ show a higher pressure, while the position at $\left(r_{\text {rel }} \mid \theta\right)=(0.83 \mid 18)$ shows a lower pressure compared to the expected circumferential average. These positions correspond to the regions of higher and lower pressure observed in Fig. 4. It is thus likely that the observed deviation between discrete three-hole probes and the circumferential average obtained from numerical simulations is caused by an inhomogeneous flow field where the measurement location directly affects the obtained value. It should be noted, however, that for the measurement positions at $\left(r_{\text {rel }} \mid \theta\right)=(0.43 \mid 18)$ and $\left(r_{\text {rel }} \mid \theta\right)=(0.71 \mid 162)$ an inverse trend between experiment and calculation can be observed.

Pressure variation is higher at the hub than at the casing, indicating that the hub region is more strongly affected. Max- imum deviation compared to the average at the hub is equal to $1.3 \%$ while it is $0.6 \%$ at the casing, which corresponds to $1292 \mathrm{~Pa}$ and $638 \mathrm{~Pa}$, respectively. The minimum deviation shows similar values, meaning that the resulting peak-to-peak amplitude equals up to $2.6 \%$ across the circumference. The maximum casing pressure is found at a circumferential position of $\theta=225 \mathrm{deg}$. Considering only the exit casing geometry, one would expect the maximum pressure to occur at a position of $\theta=90 \mathrm{deg}$. This indicates an additional dependency on swirl angle, which is equal to $\alpha=59.9 \mathrm{deg}$ at the design point. This dependency will be evaluated further in the following section by considering additional swirl angles.

In order to assess the influence on the upstream turbine section, the blade loading described by the Zweifel coefficient is depicted in Fig. 10. Similarly to the outlet pressure, blade loading also shows a total variation of $1.3 \%$ (peal-to-peak) across the circumference at midspan. Higher variation of up to $2.9 \%$ is found at the hub, while the variation amplitude decreases towards the casing. The Zweifel-number distribution shows several distinct minima and maxima rather than a continuous drop from maximum to minimum value as is the case for the trailing edge pressure, which follows a similar variation compared to that observed at the diffuser inlet. This is most likely due to the change in loading at any one passage also affecting the loading of neighboring passages because of a change in secondary flow intensity. The exit swirl causes the circumferential location of maximum loading and maximum trailing edge pressure to shift compared to the outlet distribution. The leading edge pressure variation is considerably lower than at the trailing edge. Due to the interface between the single-passage rotor and the fullannulus stator 2 domains being a mixing plane, disturbances are not convected further upstream. The effect of circumferential pressure variation on the rotor and unsteady work extraction is assumed to be considerably lower than at stator 2, but cannot be evaluated based upon the present data.

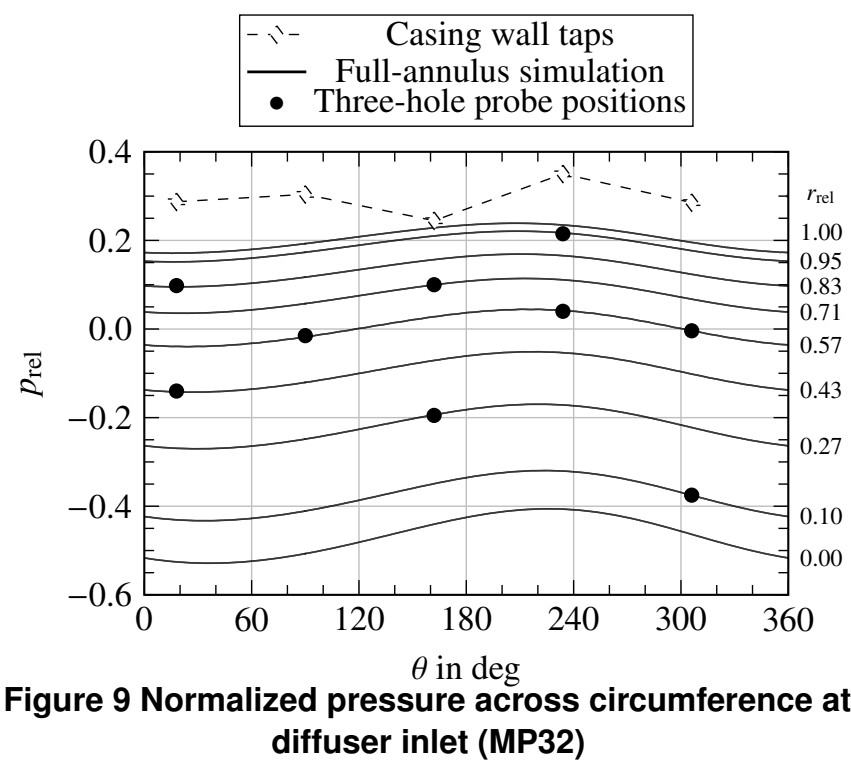




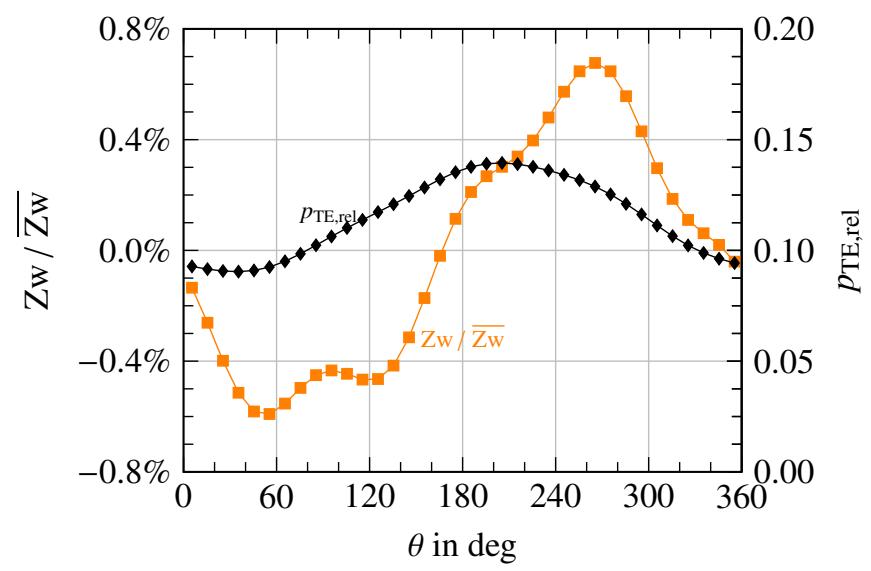

Figure 10 Circumferential distribution of Zweifel number and trailing-edge pressure at stator 2 mid-span

\section{Swirl-angle influence}

The dependence of flow inhomogeneity on swirl angle will be evaluated in this section. As mentioned, a numerical model consisting of only the exit section, i.e. diffuser and exit casing, is used for this study, allowing for independently varying the diffuser inlet swirl angle. All other parameters, i.e., diffuser inlet total state and mass flow rate, remain unchanged compared to the full-annulus calculation including the turbine section.

The swirl angle considerably affects the distortion intensity (Fig. 11). Maximum distortion occurs for a swirl angle of $\alpha=60$ deg, i.e. highly positive swirl. Highly negative swirl causes a similar flow inhomogeneity. For decreasing absolute swirl angles, the flow field returns to a more homogeneous state. Minimum distortion does not occur for a swirl angle of $\alpha=0$ deg but rather at moderate negative swirl angles in a range from $\alpha=-15 \mathrm{deg}$ to $-30 \mathrm{deg}$. Diffuser swirl strongly affects the flow separation at the discontinuous transition from diffuser to the $90 \mathrm{deg}$ bend in the exit casing. Vortex intensity increases for high absolute swirl angles, which results in a highly complex flow field within the exit casing. Moderate negative swirl angles

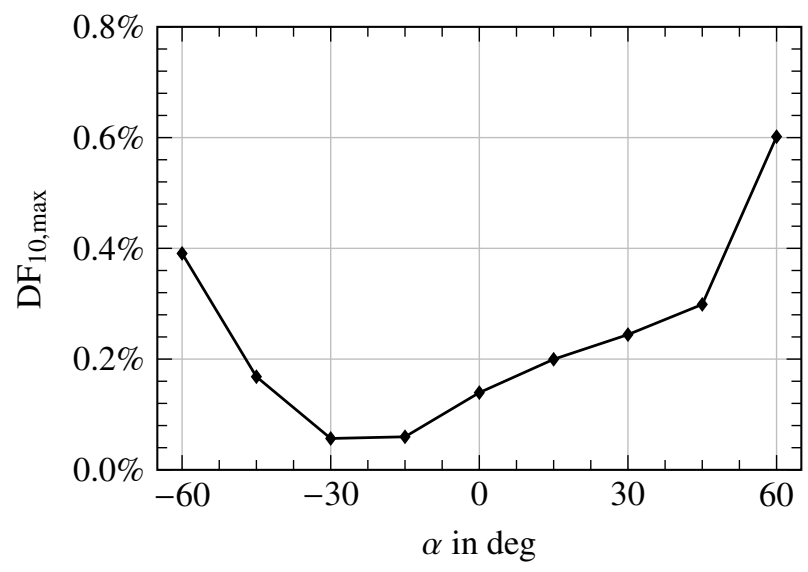

Figure 11 Swirl-angle influence on flow inhomogeneity

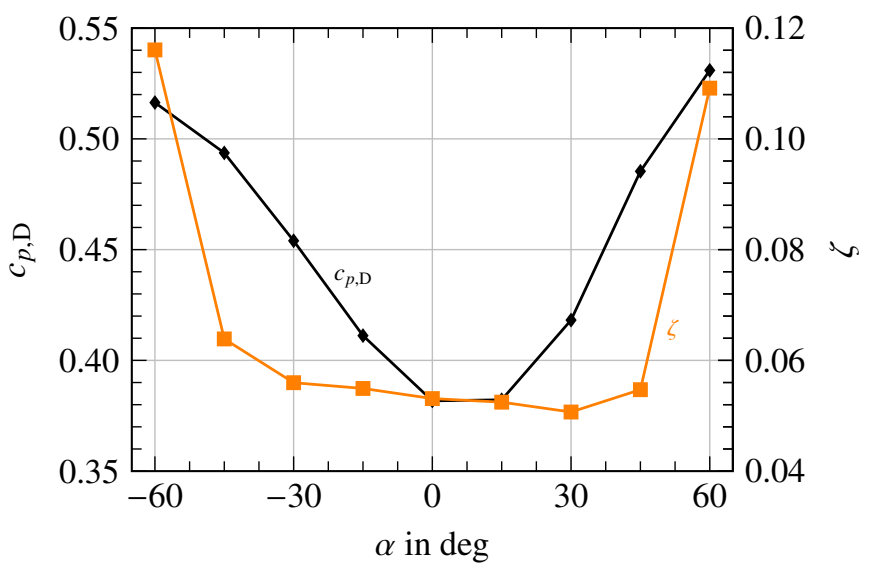
Figure 12 Swirl-angle influence on diffuser
performance

appear to have a stabilizing effect on this interaction. This is in line with the observation in Fig. 8, where the highest differences in static casing pressure are present immediately at the diffuser exit and then diminish when being transported upstream.

Finally, we consider diffuser performance in Fig. 12. For swirl angles from $\alpha=-30 \mathrm{deg}$ to $45 \mathrm{deg}$ only a slight variation in total pressure loss can be observed. Moderate positive swirl angles appear beneficial in terms of loss. If the swirl angle is increased above a certain limit, flow separation starts to occur at the diffuser hub wall. For high (absolute) swirl angles, flow separation causes a sharp increase in loss, as observed for swirl angles equal to $\alpha=-60 \mathrm{deg}$ and $\alpha=60 \mathrm{deg}$. Similarly, as the flow path length increases with higher (negative or positive) swirl angles, the diffuser further expands the fluid, causing a rise in back-pressure. $c_{p, \mathrm{D}}$ therefore increases when the swirl angle deviates from its minimum near $\alpha=0 \mathrm{deg}$. For high swirl angles, the strong increase in loss does, however, counter-act this beneficial effect.

\section{CONCLUSIONS}

Experimentally validated full-annulus calculations of a 1.5stage turbine configuration including diffuser and exit casing were conducted by deriving a simplified model. The diffuser inlet flow field was found to be homogeneous in its total quantities, the static pressure field, however, varies across the circumference. The maximum pressure occurs at a blade passage located at $\theta=225 \mathrm{deg}$ with a distortion factor of $\mathrm{DF}_{10, \max }=0.6 \%$. Evaluating the experimental casing pressure at the diffuser inlet and within the diffuser itself confirms these findings.

The flow inhomogeneity increases for higher diffuser inlet swirl angles. Other characteristic diffuser parameters depend on swirl angle as well, such as total pressure loss coefficient which increases considerably for high swirl angles inducing flow separation. We can thus conclude that the effects detailed in this paper are interdependent: While the exit casing causes inhomogeneity affecting turbine performance, the intensity is in turn dependent on the turbine outlet swirl. 
As a result of this inhomogeneity, blade loading is affected as well, deviating by up to $0.7 \%$ in Zweifel number compared to the circumferential average across the stator 2 blading. This demonstrates a moderate impact on aerodynamic performance. Depending on circumferential location, turbine measurements, such as pressure distributions obtained at individual blades, can be corrected if the local distortion factor and the exit swirl angle are known. The effect of circumferential pressure variation acting as an additional source of unsteadiness in the relative rotor frame might be even more important as it affects aeroelastic properties; potentially causing mistuning of the rotor.

These findings stress the importance of designing turbine and exit section as one system, not just due to the known impact of turbine exit flow field on diffuser performance, but also because of the exit section affecting the turbine in turn. Since the rotor was only considered as a single passage model with a mixing plane downstream, inhomogeneity influence on work extraction and rotor behavior could not be evaluated in this study. Unsteady simulations might reveal additional effects.

\section{ACKNOWLEDGMENTS}

We gratefully acknowledge the contribution of the DLR Institute of Propulsion Technology and MTU Aero Engines AG for providing TRACE.

\section{NOMENCLATURE}

\section{Latin symbols}

$\begin{array}{ll}\text { AR } & \text { aspect ratio } \\ C & \text { chord } \\ l & \text { length } \\ \dot{m} & \text { mass flow rate } \\ n & \text { rotational speed } \\ p & \text { pressure } \\ r & \text { radial coordinate } \\ T & \text { temperature } \\ x & \text { axial coordinate } \\ X & \text { axial spacing }\end{array}$

\section{Greek symbols}

$\begin{array}{ll}\alpha & \text { swirl angle } \\ \zeta & \text { loss coefficient } \\ \eta & \text { efficiency } \\ \theta & \text { circumferential coordinate } \\ \Pi & \text { pressure ratio }\end{array}$

\begin{tabular}{ll}
\multicolumn{2}{l}{ Subscripts } \\
ax & axial \\
D & diffuser \\
in & quantity at inlet \\
max & maximum quantity \\
min & minimum quantity \\
out & quantity at outlet \\
PS & pressure side \\
rel & relative quantity \\
SS & suction side \\
t & total quantity
\end{tabular}

\section{Operators}

$\overline{\text { [] }}$ averaged quantity

\begin{tabular}{ll}
\multicolumn{2}{l}{ Abbreviations } \\
CFL & Courant-Friedrichs-Levy number \\
DF & distortion coefficient \\
EC & exit casing \\
MP & measurement plane \\
R & rotor \\
Re & Reynolds number \\
S & stator \\
Zw & Zweifel number
\end{tabular}

\section{REFERENCES}

Babu, M., Bhatia, D., Shukla, R.K., Pradeep, A. and Roy, B. (2011). Effect of turbine tip leakage flows on exhaust diffuser performance. In: Proceedings of the ASME Turbo Expo, American Society of Mechanical Engineers, pp. 609-618.

Bardina, J., Ferziger, J.H. and Rogallo, R.S. (1985). Effect of Rotation on Isotropic Turbulence: Computation and Modelling. Journal of Fluid Mechanics, 154, pp. 321-336, doi: 10.1017/S0022112085001550.

Basol, A.M., Jenny, P., Ibrahim, M., Kalfas, A.I. and Abhari, R.S. (2011). Hot Streak Migration in a Turbine Stage: Integrated Design to Improve Aerothermal Performance. Journal of Engineering for Gas Turbines and Power, 133(6), doi:10.1115/1.4002349.

Biester, M.H., Henke, M., Seume, J.R., Guendogdu, Y. and Engel, K. (2012). Unsteady Wake-Blade Interaction: A Correlation Between Surface Pressure Fluctuations and Loss Generation. In: Proceedings of the ASME Turbo Expo, pp. 2743-2752, doi:10.1115/GT2012-69616.

Chernikov, V.A. (2008). A test bench for gas-dynamic investigations of exhaust diffusers and hoods of turbines. Journal of Thermal Engineering, 55(1), p. 499-505, doi: 10.1134/S0040601508060098.

Darwish, M.S. (1993). A NEW HIGH-RESOLUTION SCHEME BASED ON THE NORMALIZED VARIABLE FORMULATION. Numerical Heat Transfer, Part B: Fundamentals, 24(3), pp. 353-371, doi:10.1080/ 10407799308955898.

Feldcamp, G.K. and Birk, A.M. (2006). Strut Losses in a Diverging Annular Diffuser With Swirling Flow. In: Proceed- 
ings of the ASME Turbo Expo, vol. Volume 6: Turbomachinery, Parts A and B, pp. 9-18, doi:10.1115/GT2006-90566.

Fleige, H.U., Riess, W. and Seume, J. (2002). Swirl and Tip Leakage Flow Interaction With Struts in Axial Diffusers. In: Proceedings of the ASME Turbo Expo, doi: 10.1115/GT2002-30491.

Franke, M., Kügeler, E. and Nürnberger, D. (2005). Das DLRVerfahren TRACE: Moderne Simulationstechniken für Turbomaschinenströmungen. In: DGLR-Jahrbuch. Deutscher Luft- und Raumfahrtkongress.

Henke, M., Wein, L., Kluge, T., Guendogdu, Y., Biester, M.H. and Seume, J.R. (2016). Experimental and Numerical Verification of the Core-Flow in a New Low-Pressure Turbine. In: Proceedings of the ASME Turbo Expo, doi: 10.1115/GT2016-57101.

Kato, M. and Launder, B.E. (1993). The Modeling of Turbulent Flow Around Stationary and Vibrating Square Cylinders. In: 9th Symposium on Turbulent Shear Flows, pp. 10.4.110.4.6.

Kožulović, D., Röber, T. and Nürnberger, D. (2007). Application of a Multimode Transition Model to Turbomachinery Flows. In: Proceedings of the 7th European Conference on Turbomachinery, pp. 5-9.

Mimic, D., Drechsel, B. and Herbst, F. (2017). Correlation between Pressure Recovery of Highly Loaded Annular Diffusers and Integral Stage Design Parameters. In: Proceedings of ASME Turbo Expo 2017: Turbomachinery Technical Conference and Exposition, Charlotte, USA. Paper number GT2017-63586.

Moser, W. (1989). An Analysis of Outlet Distortion in LP Steam Turbines. In: booktitle = Proceedings of the ASME Turbo Expo,, vol. 1, doi:10.1115/89-GT-237.

Oettinger, M., Henke, M., Herbst, F. and Seume, J.R. (2019a). Axial-Gap Size Effect on the Unsteady Flow Field at Midspan in a 1.5-Stage Low-Pressure Turbine. In: Proceedings of International Gas Turbine Congress Tokyo, doi: IGTC2019-0084.

Oettinger, M., Mimic, D., Henke, M., Oleg, S. and Seume, J.R. (2019b). Loss Assessment of the Axial-Gap Size Effect in a Low-Pressure Turbine. In: Proceedings of Global Power and Propulsion Society Beijing Conference, doi: GPPS-BJ-2019-0207.

Opilat, V. (2011). Swirl and Blade Wakes in the Interaction between Gas Turbines and Exhaust Diffusers Investigated by Endoscopic Particle Image Velocimetry. PhD Thesis, Leibniz Universität Hannover, Hanover.

Pradeep, A.M., Roy, B., Vaibhav, V. and Srinuvasu, D. (2010). Study of Gas Turbine Exhaust Diffuser Performance and Its Enhancement by Shape Modifications. In: Proceedings of the ASME Turbo Expo, vol. 7, pp. 1101-1110, doi:10.1115/ GT2010-22088.

Pyliouras, S., Schiffer, H.P., Janke, E. and Willer, L. (2012). Effects of Non-Uniform Combustor Exit Flow on Turbine Aerodynamics. In: Proceedings of the ASME Turbo Expo, vol. 8, pp. 1691-1701, doi:10.1115/GT2012-69327.

Sideris, M.T. and Van den Braembussche, R.A. (1987). Influence of a Circumferential Exit Pressure Distortion on the Flow in an Impeller and Diffuser. Journal of Turbomachinery, 109(1), pp. 48-54, doi:10.1115/1.3262069.

Simonassi, L., Zenz, M., Bruckner, P., Heitmeir, F. and Marn, A. (2019). Aeroelastic and Aerodynamic Investigation of a Low Pressure Turbine Under the Influence of a Circumferential Inlet Distortion. In: Proceedings of the ASME Turbo Expo, vol. 7, doi:10.1115/GT2019-90887.

van Albada, G.D., van Leer, B. and Roberts, Jr., W.W. (1982). A Comparative Study of Computational Methods in Cosmic Gas Dynamics. Astron Astrophys, 108, pp. 76-84.

Vassiliev, V., Irmisch, S., Abdel-Wahab, S. and Granovskiy, A. (2011). Impact of the Inflow Conditions on the HeavyDuty Gas Turbine Exhaust Diffuser Performance. Journal of Turbomachinery, 134(4), doi:10.1115/1.4003714.

Vassiliev, V., Rothbrust, M. and Irmisch, S. (2008). Refitting of Exhaust Diffuser of Industrial Gas Turbine. In: Proceedings of the ASME Turbo Expo, vol. 6, pp. 979-987, doi:10.1115/ GT2008-50165.

Volkmer, S., Hirschmann, A., Casey, M. and Montgomery, M. (2011). The Impact of a Tip Leakage Jet on Flow Separation in Axial Gas Turbine Diffusers. In: European Turbomachinery Conference, Istanbul, Turkey.

Wilcox, D.C. (1998). Turbulence Modeling for CFD. 2nd edn., DCW Industries. 